\title{
The diagnostic role of cardiac magnetic resonance used "first and last time in life" in a patient with a suspected dilated phase of hypertrophic cardiomyopathy
}

\author{
Rafał Hładij, Renata Rajtar-Salwa, Artur Dziewierz, Paweł Petkow-Dimitrow \\ $2^{\text {nd }}$ Department of Cardiology, Jagiellonian University Medical College, Krakow, Poland
}

Adv Interv Cardiol 2017; 13, 2 (48): 178-179

DOI: https://doi.org/10.5114/pwki.2017.68049

A 38-year-old female patient with neither diagnosis nor family history of any heart disease was admitted to the regional hospital with sustained ventricular tachycardia (220 bpm). Tachyarrhythmia was successfully treated with intravenous amiodarone. Total time of sustained ventricular tachycardia was no more than $1 \mathrm{~h}$ (from the start of symptoms at home to the end of arrhythmia in the emergency department in a regional hospital). The pulmonary congestion was revealed both in physical and $X$-ray examination. Extremely high levels of high-sensitivity troponin I (> $40000 \mathrm{ng} / \mathrm{l}$ ) as well as NT-proBNP (21 $055 \mathrm{pg} / \mathrm{ml}$ ) were detected. In control sampling after several days the level of troponin I was still very high: $39185 \mathrm{ng} / \mathrm{l}$. The patient was transferred to our tertiary center where in the face of a prolonged period of an extremely high level of troponin I, the decision to perform coronary angiography was made. The coronary artery angiogram was normal. Echocardiography revealed myocardial hypertrophy and mild dilatation of the left ventricle (LV). The LV ejection fraction (EF) was low, i.e. $28 \%$. The suspicion of the starting phase of the dilative stage (burn-out) of hypertrophic cardiomyopathy (HCM) was suggested and cardiac magnetic resonance (CMR) imaging with gadolinium contrast was proposed to explore the hypothesis.

The CMR confirmed myocardial hypertrophy distributed in several segments. Maximal thickness of the myocardium was $19 \mathrm{~mm}$ in diastole. Total LV mass was increased to $303 \mathrm{~g}\left(156 \mathrm{~g} / \mathrm{m}^{2}\right)$.

Mild LV cavity dilatation was characterized by the following parameters: end diastolic volume of $318 \mathrm{ml}$ $\left(163 \mathrm{ml} / \mathrm{m}^{2}\right)$ and end systolic volume of $271 \mathrm{ml}(112 \mathrm{ml}$ $\mathrm{m}^{2}$ ). Low EF was confirmed at the level of $30 \%$. A large amount $(29.6 \%)$ of late gadolinium enhancement (LGE) with a diffused pattern was noted (Figure 1).
An episode of sustained ventricular tachycardia has been recognized as a major risk factor of sudden cardiac death (SCD). Therefore, an implantable cardioverter-defibrillator with resynchronization (R-ICD) was implanted. This decision was supported by a wide QRS (130 ms) complex and NYHA class defined as III during hospitalization. Resynchronization was introduced due to low LV EF. The decision of electrotherapy as secondary prevention of SCD was supported by a massive amount of LGE representing the arrhythmia substrate. Unfortunately, R-ICD implantation will not allow follow-up examinations with CMR to be made in the future.

In our case the large amount of LGE was associated with lower EF. Thus, the heart was probably in an initial phase of evolution to the dilated end-stage (burning out) phase which occurs in $5-10 \%$ of patients with HCM [1].

One of the strongest and most consistent observations derived from a number of contemporary cross-sectional studies is the inverse relationship between EF and LGE in HCM. The LGE extent is greatest in HCM patients with LV EF $<50 \%$ (heralding the development of the burn-out phase) [1].

In a recent study on patients undergoing transplantation (excised heart) due to end-stage HCM, more than one-third of the LV myocardium of those patients was replaced by fibrosis [2]. In our patient a similar value of LGE (near 30\%) was detected.

In a very recent meta-analysis, quantitative LGE by CMR exhibited a substantial and independent of baseline characteristics value in the prediction of SCD. Thus, assessment of LGE can be used as an effective tool for risk stratification of patients with HCM [3]. An LGE value > 15\% has been recognized as a strong risk factor for ventricular fibrillation.

An increased (even persistently) level of troponin in HCM has been described in recently published papers.

\section{Corresponding author:}

Paweł Petkow-Dimitrow MD, PhD, $2^{\text {nd }}$ Department of Cardiology, Jagiellonian University Medical College, 17 Kopernika St, $31-501$ Krakow, Poland, phone: +48 12424 7170, e-mail: dimitrow@mp.pl

Received: 19.11.2016, accepted: 11.01.2017. 


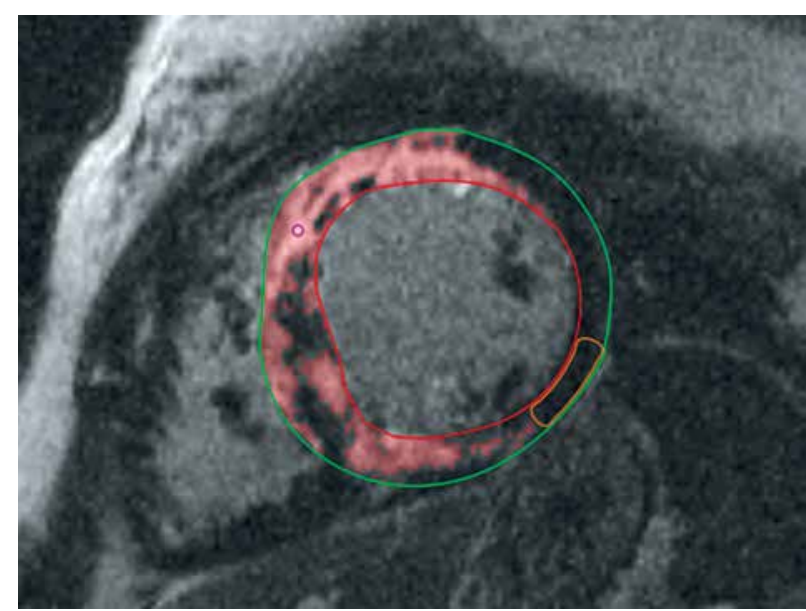

Figure 1. Massively diffused late enhancement visualized in short axis imaging

The scale of the phenomenon is large [4] as a positive test for high-sensitivity troponin $\mathrm{T}$ was detectable in $3 / 4$ and elevated in $1 / 4$ of patients with HCM. In obstructive $\mathrm{HCM}$, troponin I was also shown to be correlated with the extent of LV hypertrophy [5]. In addition, this biomarker was an independent predictor of the presence of myocardial fibrosis in CMR [6]. Recently, we reported an increased level of troponin after supraventricular tachycardia $180 \mathrm{bpm}$ in a patient with apical HCM [7]. This observation as well as the present case suggests that tachycardia may be a trigger for troponin release in patients with HCM.

Importantly, from a therapeutic point of view, it has been documented in HCM [8] that increased troponin release may be present in such patients and may be temporarily enhanced by exercise and decreased with $\beta$-blockers. Possibly in our patient sustained ventricular tachycardia may have mimicked very extensive exercise and may have been responsible for a huge release of troponin.

In conclusion, CMR imaging may be helpful as a diagnostic tool in patients with life-threatening sustained ventricular tachyarrhythmia (without a history of any heart disease) and an ambiguous echocardiographic picture. Extremely high levels of troponin I despite a normal coronary artery angiogram may indicate development of the burn-out phase of the disease, called the dilative form of HCM.

\section{Conflict of interest}

The authors declare no conflict of interest.

\section{References}

1. Maron MS. Clinical utility of cardiovascular magnetic resonance in hypertrophic cardiomyopathy. J Cardiovasc Magn Reson 2012; 14: 13.

2. Galati G, Leone O, Pasquale F, et al. Histological and histometric characterization of myocardial fibrosis in end-stage hyper- trophic cardiomyopathy: a clinical-pathological study of 30 explanted hearts. Circ Heart Fail 2016; 9: pii: e003090.

3. Briasoulis A, Mallikethi-Reddy S, Palla M, et al. Myocardial fibrosis on cardiac magnetic resonance and cardiac outcomes in hypertrophic cardiomyopathy: a meta-analysis. Heart 2015; 101: 1406-11.

4. Cramer G, Bakker J, Gommans F, et al. Relation of highly sensitive cardiac troponin $\mathrm{T}$ in hypertrophic cardiomyopathy to left ventricular mass and cardiovascular risk. Am J Cardiol 2014; 113: 1240-5.

5. Zhang C, Liu R, Yuan J, et al. Predictive values of N-terminal proB-type natriuretic peptide and cardiac troponin I for myocardial fibrosis in hypertrophic obstructive cardiomyopathy. PLoS One 2016; 11: e0146572.

6. Zhang C, Liu R, Yuan J, et al. Significance and determinants of cardiac troponin I in patients with obstructive hypertrophic cardiomyopathy. Am J Cardiol 2015; 116: 1744-51.

7. Rajtar-Salwa, Petkow-Dimitrow P, Miszalski-Jamka T. Role of cardiac magnetic resonance in differentiating between acute coronary syndrome and apical hypertrophic cardiomyopathy. Adv Interv Cardiol 2016; 12: 380-2.

8. Pop GA, Cramer E, Timmermans J, et al. Troponin I release at rest and after exercise in patients with hypertrophic cardiomyopathy and the effect of betablockade. Arch Cardiol Mex 2006; 76: 415-8. 